\title{
EFECTOS SOBRE LA ERD EN TAREAS DE CONTROL DE EXOESQUELETO DE MANO EMPLEANDO BCI
}

\author{
S. Ezquerro, Juan A. Barios, A. Bertomeu-Motos, L. Lorente, N. Requena, \\ I. Delegido, Francisco J. Badesa, N. Garcia-Aracil \\ Universidad Miguel Hernández, Elche (Alicante) 03202, \\ abertomeu@umh.es
}

\section{Resumen}

Los estudios con interfaces cerebro-máquina basados en electroencefalografía (EEG) en el campo de la rehabilitación y ayuda a personas con movilidad reducida, típicamente basados la modulación de los ritmos sensoriomotores (SMR), han aumentado notablemente en estos últimos diez años. Unidos a la aparición de nuevas herramientas en rehabilitación, como son los exoesqueletos, prometen ser una poderosa alternativa terapéutica y rehabilitadora para la recuperación motora de las lesiones distales de los miembros superiores. Diez sujetos sanos han participado en este estudio, en el cual se cuantificó en tiempo real las modulaciones de los ritmos SMR en la banda alpha $(8-12 \mathrm{~Hz})$ durante una actividad de de imaginería motora (IM) (cierre de mano/relax) utilizando diferentes modalidades sensoriales como feedback: propioceptivo (utilizando un exoesqueleto de mano), auditivo y visual. Los resultados obtenidos muestran una incremento en la intensidad y la duración de la desincronización (ERD) en la banda alpha durante la IM utilizando el exoesqueleto.

Palabras clave: Brain-computer interfaces, electromiography, motor imagery, exoskeleton.

\section{INTRODUCCIÓN}

El desarrollo de tecnología BCI (Brain-Computer Interface) basada en EEG (electroencefalografía) ha aumentado notoriamente en la última década en campos como la rehabilitación [1], entretenimiento o dirigidos a personas con discapacidad para la realización de las actividades de la vida diaria (AVDs) [2].

A través de BCI basado en EEG podemos observar la modulación de los ritmos sensoriomotores (SMRs), estos ritmos se registran en el área sensoriomotora primaria (M1) y son oscilaciones que ocurren en las bandas alpha $(8-12 \mathrm{~Hz})$ y beta (13$35 \mathrm{~Hz}$ ). Una de las características principales de esta área es que su amplitud disminuye durante el movimiento y es similar cuando se repite el movimiento mentalmente a través de la Imaginería
Motora (IM) [3] [4]. Diferentes estudios han mostrado que las personas pueden aprender a modular la amplitud del SMR mediante la práctica de movimientos sencillos (mano o pie) a través de la IM [5] [6]. Este proceso se produce en bucle cerrado, es decir, el sistema reconoce los cambios de amplitud del SMR provocados por la IM y la respuesta es enviada al usuario en forma de feedback visual. Este procedimiento y la adaptación humano-máquina permite a los usuarios de BCI controlar su actividad SMR y utilizar estas modulaciones para controlar dispositivos de salida de manera asíncrona [7].

El objetivo principal de este estudio fue observar las modulaciones del SMR mediante la desincronización (ERD) o sincronización (ERS) de los potenciales en la banda alpha producidos en la corteza sensoriomotora primaria (M1) mediante tareas de imaginería motora (IM) empleando como instrumentos un sistema de BCI y un exoesqueleto de mano.

\section{MATERIAL Y MÉTODOS}

\subsection{Participantes}

Diez sujetos sanos han participado en este estudio durante 5 sesiones a lo largo de cinco semanas consecutivas con duración de 90 minutos por cada una. Los criterios de inclusión propuestos para este estudio fueron: adultos sanos sin ningún tipo de déficit cognitivo diagnosticado y diestros, debido a que el exoesqueleto empleado era para mano derecha. Los criterios de exclusión fueron aquellos que contradecían a los anteriores. Todos los participantes firmaron el consentimiento informado.

\subsection{Diseño experimental}

Las experimentaciones se realizaron en una sala tranquila y controlada. Todos los participantes se sentaron frente a una pantalla, la cual se empleaba para dar las órdenes de movimiento. El EEG se registró en cinco áreas $(\mathrm{F} 3, \mathrm{C} 3, \mathrm{CZ}, \mathrm{T} 3 \mathrm{Y}$ P3, de acuerdo con el sistema internacional 10/20) y empleando un gorro EEG de neopreno (Neuroelectrics $\left.{ }^{\circledR}\right)$, colocando como electrodos de referencia 
Fz y C4 (Figura 1). La electrooculografía (EOG) fue registrada en concordancia a la localización estándard para EOG, en el canto externo de los ojos (LOC). El EEG se registró con una frecuencia de muestro de $200 \mathrm{~Hz}$, y se realizó un filtrado paso banda entre $0.5-40 \mathrm{~Hz}$.

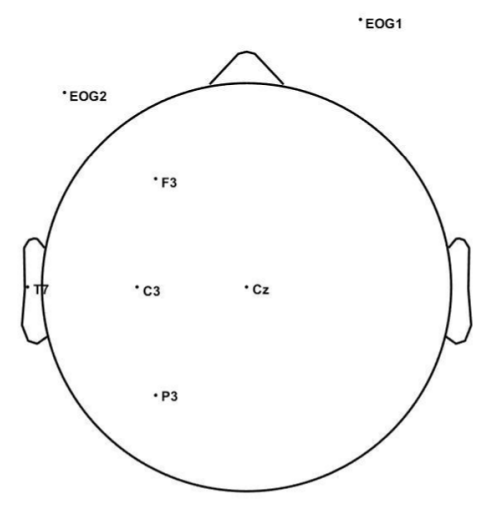

Figura 1: Esquema colocación de los electrodos en el encéfalo.

Para esta experimentación se empleó una versión de BCI2000, una plataforma multiuso estándar [8], para la calibración y control online del BNCI. Esta calibración constaba de dos partes: (i) Horizontal electrooculography (HEOG), se registraron datos de los movimientos oculares de los sujetos (derecha o izquierda), con este registro se calculó la diferencia de potencial entre la córnea y la retina para emplearla como señal de aborto de un movimiento (Figura 2); (ii) Threshold relax-close (THRC), mediante una tarea de cierre, imaginando el movimiento de agarre, y una tarea de relax, se tomaron los valores de los ritmos sensoriomotores en la corteza motora izquierda. Estas instrucciones aparecían en la pantalla del ordenador de forma alternante. Estas indicaciones estaban separadas por un intervalo entre ensayos de unos 4-6 segundos (Figura 3).
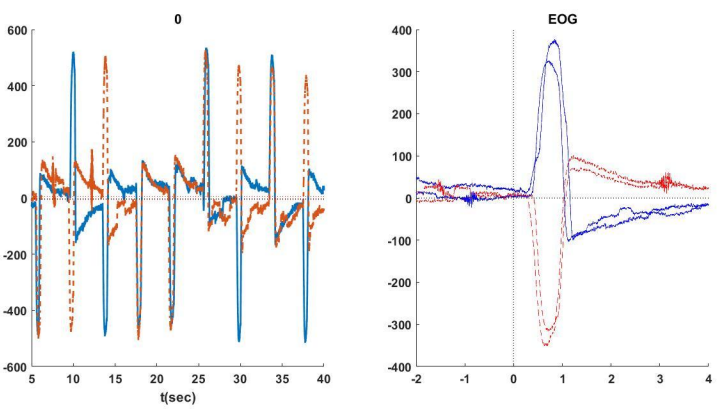

Figura 2: Ejemplo HEOG de un usuario después de la calibración.

Para facilitar al usuario la tarea de imaginería motora, se le presenta un feedback visual, en este caso
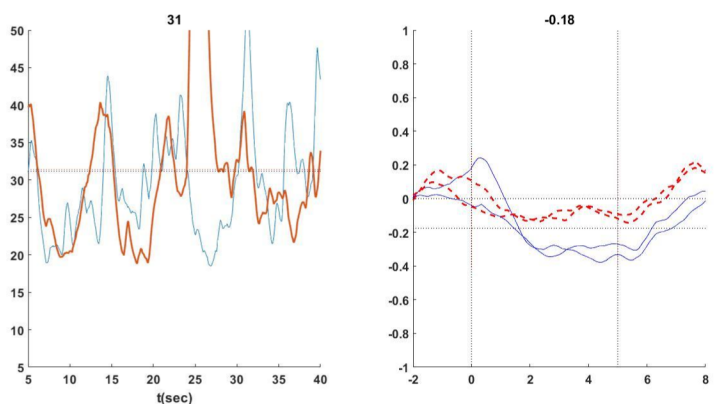

Figura 3: Ejemplo THRC de un usuario después de la calibración donde la línea continua corresponde al cierre (IM) y la línea discontinua al relax.

era la figura "Pac-Man", la cual comenzaba a cerrarse cuando el sujeto pensaba en la acción de cerrar la mano.

\subsubsection{Exoesqueleto}

El dispositivo es un robot tipo exoesqueleto con tres grados de libertad, que puede actuar de forma independiente sobre los dedos índice, corazón y el grupo anular-meñique. El movimiento de los dedos indicados se controla por medio de un mecanismo de barras que acopla las rotaciones de las falanges proximal y medial. Adicionalmente, el dispositivo permite fijar la posición del dedo pulgar mediante un mecanismo de barras regulable con opción de bloqueo. Este mecanismo se fija a las falanges proximales y mediales de cada dedo mediante unos anillos elásticos que se insertan por presión en el mecanismo (Figura 4).

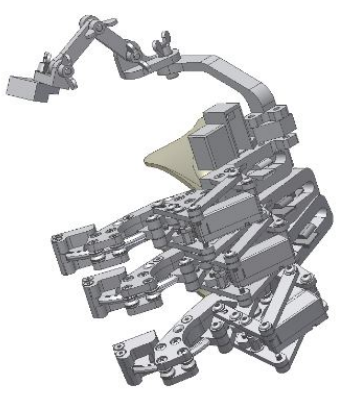

Figura 4: Diseño del exoesqueleto de mano.

A su vez, la mano se fija al bastidor mediante una órtesis ajustable por correas. En la tabla 1 se pueden observar las especificaciones técnicas del exoesqueleto.

\subsection{Procesamiento de datos}

Para el procesamiento y análisis de los datos EEG, se empleó la herramienta de Matlab, EEGLAB [9]. 
Tabla 1: Especificaciones técnicas del exoesqueleto de mano empleado en la experimentación.

\begin{tabular}{|c|c|c|}
\hline \multicolumn{3}{|c|}{ Conjunto motor - Índice } \\
\hline Potencia & 2.52 & {$[\mathbf{W}]$} \\
\hline Reducción & 100:1 & Adim \\
\hline Fuerza máxima & 50 & {$[\mathbf{N}]$} \\
\hline Recorrido angular (MCP) & $10 \div 45$ & [grados] \\
\hline Recorrido angular (PIP) & $15 \div 70$ & [grados] \\
\hline \multicolumn{3}{|c|}{ Conjunto motor - Corazón } \\
\hline Potencia & 2.52 & {$[\mathbf{W}]$} \\
\hline Reducción & 100:1 & Adim \\
\hline Fuerza máxima & 50 & {$[\mathbf{N}]$} \\
\hline Recorrido angular (MCP) & $10 \div 45$ & [grados] \\
\hline Recorrido angular (PIP) & $15 \div 70$ & [grados] \\
\hline \multicolumn{3}{|c|}{ Conjunto motor - Anular y Meñique } \\
\hline Potencia & 2.52 & {$[\mathbf{W}]$} \\
\hline Reducción & 100:1 & Adim \\
\hline Fuerza máxima & 50 & {$[\mathbf{N}]$} \\
\hline Recorrido angular (MCP) & $10 \div 45$ & [grados] \\
\hline Recorrido angular (PIP) & $15 \div 70$ & [grados] \\
\hline
\end{tabular}

Se realizó una serie de pasos de pre-procesado de los datos obtenidos. En primer lugar, se realizó un paso banda en la frecuencia $0.5 \mathrm{H}$. y un remuestreado de 500 a $128 \mathrm{~Hz}$. El siguiente paso fue extraer las épocas de cada uno de los ensayos, para ello, subdividimos los intervalos en 11 segundos, 3 segundos pre-estímulo de la tarea y 8 segundos después del estímulo. De este modo, ambas condiciones pudieron ser comparadas. El punto de referencia (tiempo cero) fue asignado al comienzo del estímulo visual. La longitud de los intervalos, antes y después del punto de referencia, fueron escogidos como la longitud de los MRCP (aproximadamente 2 segundos). Una vez subdivididos en épocas se analizaron los posibles artefactos.

Se limitó el rango de frecuencia entre 0.5-48 Hz, así como, el periodo de tiempo en un rango de 3 segundos antes de la orden visual y 8 segundos después, tres segundos después de la orden marcando el final de la tarea. La longitud de las épocas EEG (de 3 a 8 segundos) abarcó puntos de tiempo más allá del período de interés, a fin de incluir una señal suficiente antes y después de los bordes de ese período de interés (0 y 5 segundos relativos al comienzo de la tarea). El rango de frecuencia que hemos incluido para todas las bandas de frecuencia del EEG fueron: $\delta(1-3 \mathrm{~Hz}), \theta(4-7 \mathrm{~Hz}), \alpha$ (8-12 Hz), $\beta$ (13-30) y $\gamma(30-48 \mathrm{~Hz})$. Para el análisis estadístico, los métodos estaban incluidos en el mismo paquete de trabajo que se ha empleado.

\section{RESULTADOS}

\subsection{Umbral relax-cierre (THRC)}

En primer lugar, se analizaron los resultados de la calibración de la prueba THRC. Durante este ensayo, los sujetos a través de IM tenían que imaginar el movimiento de cierre de la mano. En la figura 5 se observan los cambios en el espectro de los eventos relacionados (ERSP) producidos por las modulaciones del SMR de todos los sujetos en C3 durante las cinco sesiones en la orden de cierre. Se observa una desincronización del potencial en la banda $\alpha$ (8-12 Hz) en todos los ensayos, pronunciándose cada vez más a lo largo del tiempo. Por otro lado, en la banda $\theta(4-8 \mathrm{~Hz})$ se visualiza un aumento del potencial a lo largo de las sesiones.

\subsection{Umbral con exoesqueleto}

Una vez calibrado el sistema BCI y entrenados los sujetos, se registró actividad rítmica sensoriomotora durante el uso del exoesqueleto de mano. En la figura 6 se observan los cambios sensoriomotores (SMR) en la banda $\alpha(8-12 \mathrm{~Hz})$, tanto en la orden de cierre como en la de relax. El espectro de la figura muestra una desincronización (ERD) en la banda alpha cuando el sujeto piensa en el movimiento de cierre de mano y se mantiene hasta el final de la orden. Mientras que en la tarea de relax (derecha), se observa que durante esta orden se produce una sincronización (ERS) en esta misma banda, es decir, el potencial se mantiene durante todo el tiempo.

En la Figura 7 se observan los cambios del SMR que se producen en los sujetos durante la actividad de IM (izquierda) y de relax (derecha), pero en esta prueba el sujeto no llevaba puesto el exoesqueleto, con estos resultados queríamos comprobar que efectos se producían en las modulaciones del SMR cuando al sujeto se le proporcionaba además de un estímulo visual (orden en la pantalla), un estímulo auditivo (sonido del exoesqueleto cerrando y abriéndose). Como en la anterior figura se puede visualizar que ocurren cambios en los potenciales de la banda alpha. No obstante, en la tarea de IM podemos observar que esta desincronización tiene una duración en el tiempo menor que en el caso anterior, volviendo a sincronizarse poco antes de los 4 segundos. En cuanto al relax, sucede lo mismo que en el caso anterior, los sujetos son capaces de mantener sincronizados los ritmos en la banda alpha.

\section{DISCUSIÓN}

Tras el análisis estadístico realizado con los datos obtenidos de los ensayos con BCI basados en 

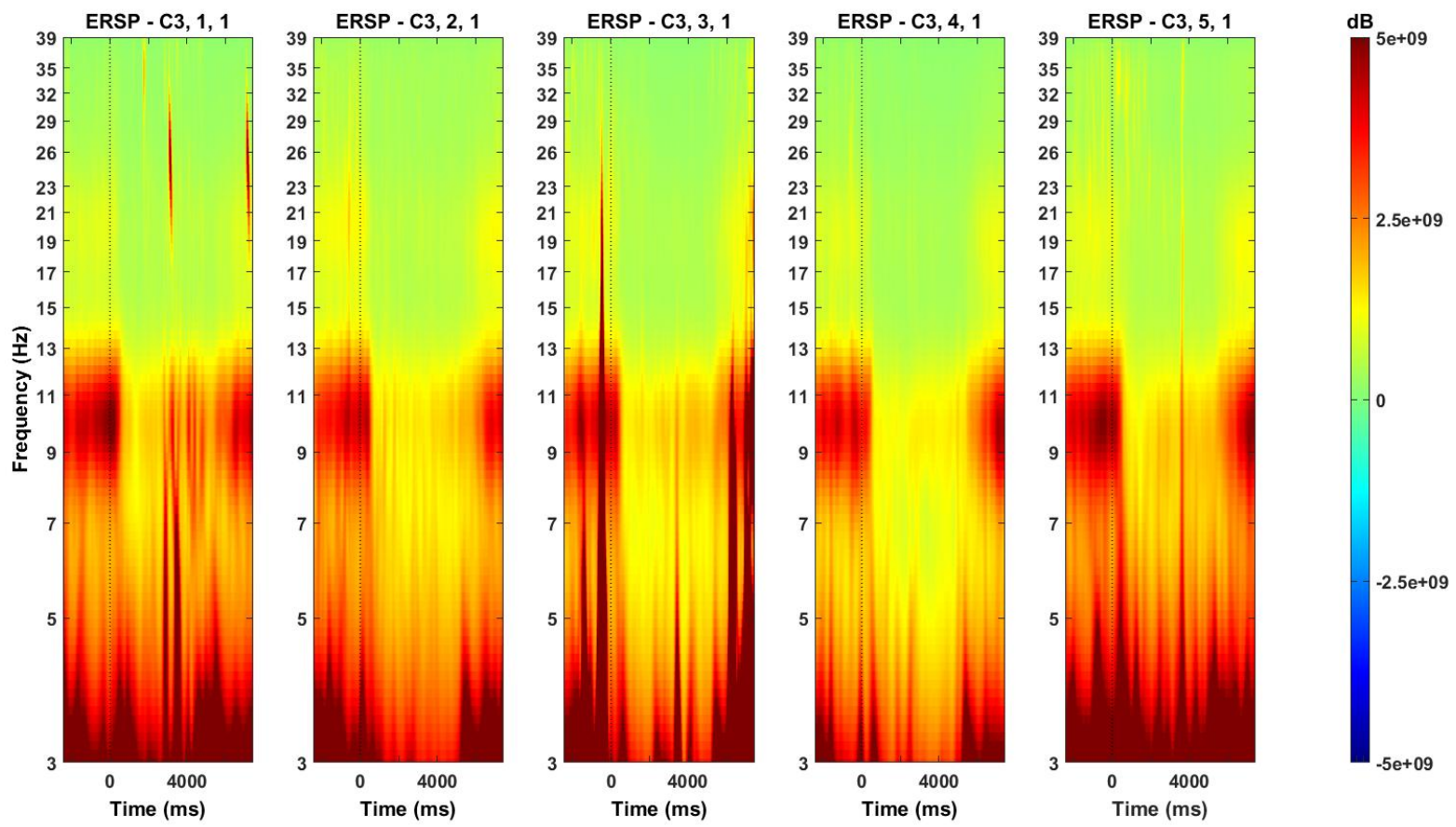

Figura 5: Modulaciones del SMR a lo largo de las cinco sesiones de entrenamiento de IM.
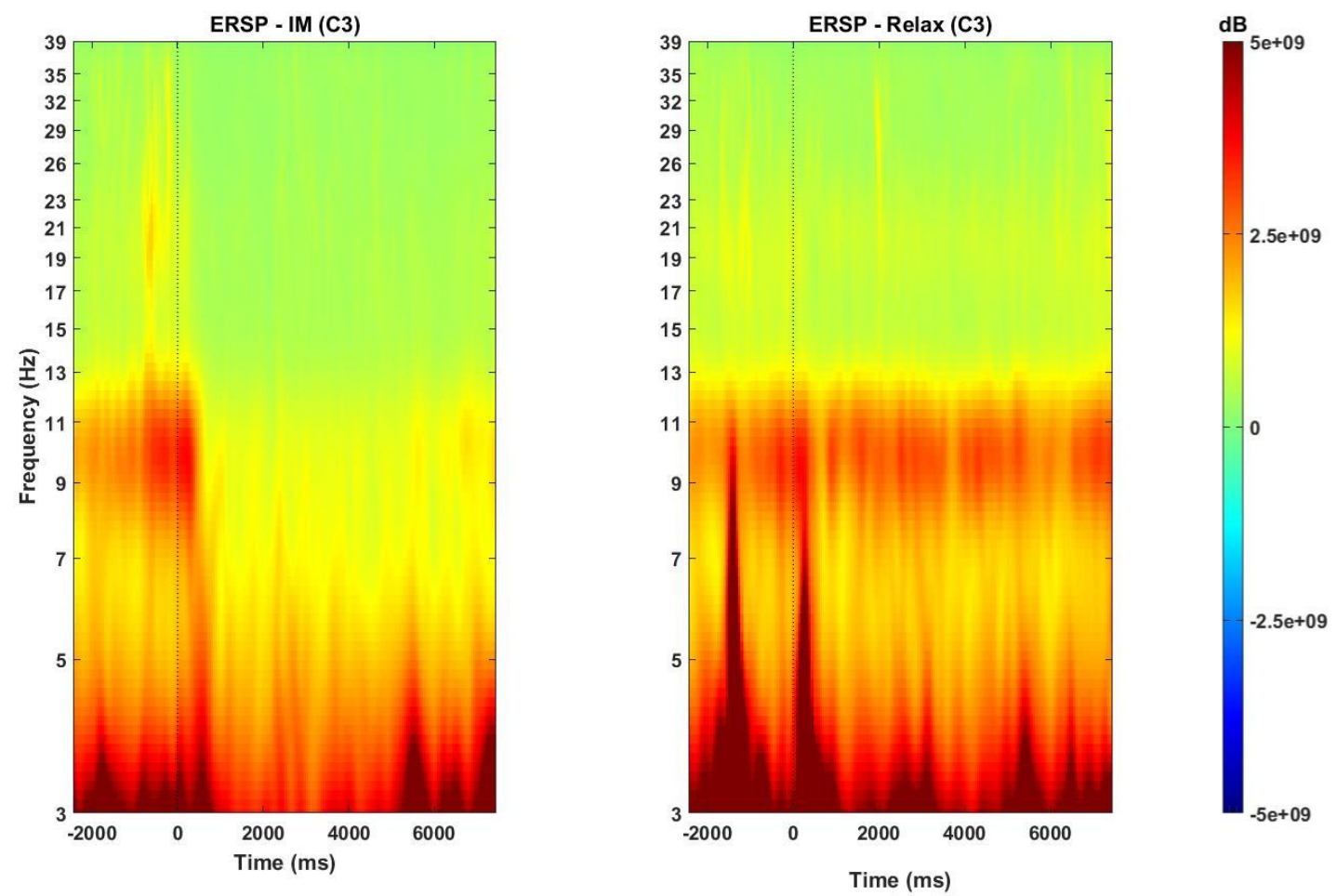

Figura 6: Cambios en el SMR durante la actividad de cierre por IM (izquierda), la actividad de relax (derecha) con el exoesqueleto colocado. 

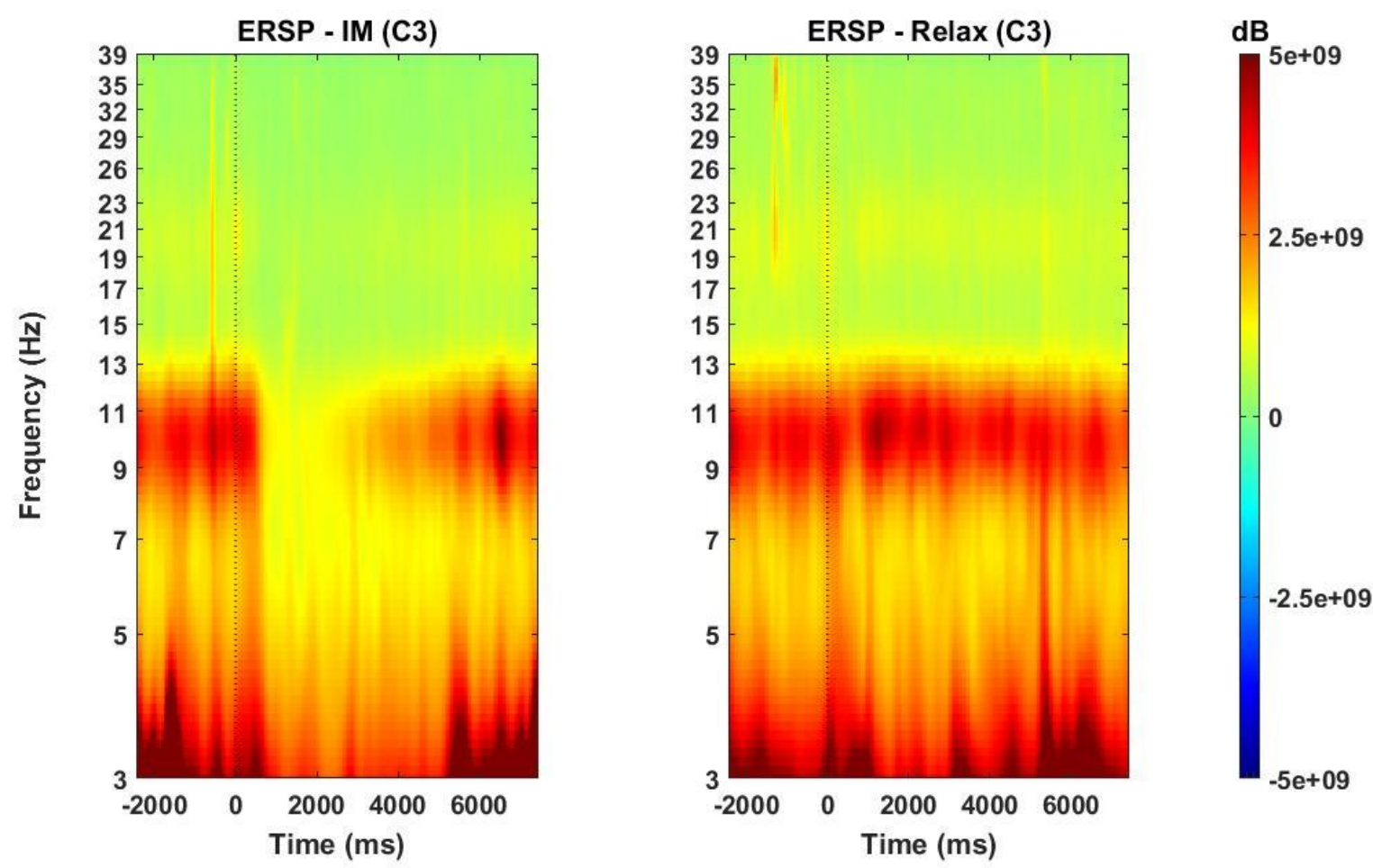

Figura 7: Cambios en el SMR durante la actividad de cierre por IM (izquierda), la actividad de relax (derecha) sin el exoesqueleto.

electromiografía y la literatura revisada para este ensayo podemos determinar que existe una activación de potenciales en banda alpha $(8-12 \mathrm{~Hz})$ en la corteza sensoriomotora primaria cuando imaginamos un movimiento de cierre de mano provocando así una ERD en dicha banda y volviendo a sincronizar en torno a los 5-6 segundos (fin de la orden visual). Así mismo esta ERD se hace más consistente con el entrenamiento en IM. También podemos observar un aumento de potencial en los ritmos lentos en la banda theta $(4-7 \mathrm{~Hz})$, lo que nos indica una inhibición de dichos ritmos. Estos datos nos revelan que existe una modulación en los cambios de potenciales y un aumento de desincronización en tareas de movimiento motor lo que nos indica un aprendizaje de imaginería motora por parte de los sujetos.

En cuanto a los resultados obtenidos en el entrenamiento con el exoesqueleto de mano, podemos observar que en ambos casos (entrenamiento con estímulo propioceptivo y con estímulo auditivo) se produce una ERD cuando el sujeto piensa en el movimiento de agarre a través de IM. No obstante, el tiempo de activación del SMR durante el entrenamiento con estímulo auditivo (sin exoesqueleto) es menor que si se le añade al entrenamiento un estímulo propioceptivo (con exoesqueleto), lo que nos lleva a pensar que el entrenamiento con el exoesqueleto puesto se ve reforzado por dicho estímulo.

\section{CONCLUSIONES}

Durante el cierre voluntario del exoesqueleto mediante IM, hemos observado una desincronización en el SMR en la banda alpha que refleja la la activación de la corteza sensoriomotora M1 a través de IM de un movimiento de cierre de mano. También hemos observado que estos ritmos se modulan con el tiempo con la progresión del entrenamiento de esta técnica, con una intensidad dependiente de la modadlidad sensorial utilizada, lo que podría llegar a ser una buena herramienta en la rehabilitación de personas con algún tipo de déficit motor causado por alguna patología de origen traumatológica o neurológica.

\section{Agradecimientos}

Este trabajo ha sido financiado por la Comisión Europea a través del proyecto AIDE: Adaptive Multimodal Interfaces to Assist Disabled People in Daily Activities (Grant agreement no: 645322) y por el Ministerio de Economía y Competitividad a través del proyecto DPI2015-70415-C2-2-R. 


\section{Referencias}

[1] J. J. Daly and J. R. Wolpaw, "Brain-computer interfaces in neurological rehabilitation," The Lancet Neurology, vol. 7, no. 11, pp. 1032 1043, 2008.

[2] G. Pfurtscheller, C. Brunner, A. Schlögl, and F. L. Da Silva, "Mu rhythm (de) synchronization and eeg single-trial classification of different motor imagery tasks," NeuroImage, vol. 31, no. 1, pp. 153-159, 2006.

[3] G. Pfurtscheller and F. L. Da Silva, "Eventrelated eeg/meg synchronization and desynchronization: basic principles," Clinical neurophysiology, vol. 110, no. 11, pp. 1842-1857, 1999.

[4] C. Neuper, R. Scherer, M. Reiner, and G. Pfurtscheller, "Imagery of motor actions: Differential effects of kinesthetic and visual-motor mode of imagery in single-trial eeg," Cognitive brain research, vol. 25, no. 3, pp. 668-677, 2005.

[5] V. Kaiser, G. Bauernfeind, A. Kreilinger, T. Kaufmann, A. Kübler, C. Neuper, and G. R. Müller-Putz, "Cortical effects of user training in a motor imagery based braincomputer interface measured by fnirs and eeg," Neuroimage, vol. 85, pp. 432-444, 2014.

[6] J. Toppi, M. Risetti, L. Quitadamo, M. Petti, L. Bianchi, S. Salinari, F. Babiloni, F. Cincotti, D. Mattia, and L. Astolfi, "Investigating the effects of a sensorimotor rhythm-based bci training on the cortical activity elicited by mental imagery," Journal of neural engineering, vol. 11, no. 3, p. 035010, 2014.

[7] J. A. Pineda, D. S. Silverman, A. Vankov, and J. Hestenes, "Learning to control brain rhythms: making a brain-computer interface possible," IEEE transactions on neural systems and rehabilitation engineering, vol. 11, no. 2, pp. 181-184, 2003.

[8] G. Schalk, D. J. McFarland, T. Hinterberger, N. Birbaumer, and J. R. Wolpaw, "Bci2000: a general-purpose brain-computer interface (bci) system," IEEE Transactions on biomedical engineering, vol. 51, no. 6, pp. 1034-1043, 2004.

[9] A. Delorme and S. Makeig, "Eeglab: an open source toolbox for analysis of single-trial eeg dynamics including independent component analysis," Journal of neuroscience methods, vol. 134, no. 1, pp. 9-21, 2004. 